\title{
How to Link Accident Data and Road Traffic Measurements to Enable ADAS/AD Simulation?
}

\author{
Christian T. Erbsmehl ${ }^{1}$, Thomas Lich $^{2}$ and Jorge Mallada ${ }^{3}$ \\ 1. Vehicle and Road Safety, Fraunhofer IVI, Dresden 01067, Germany \\ 2. Research Accident, Robert Bosch GmbH, Stuttgart 70465, Germany \\ 3. Safety Research \& Technical Affairs, Toyota Motor Europe, Zaventem 1930, Belgium
}

\begin{abstract}
The progress of safety technologies, based on the continuous advances in vehicle crash worthiness, restraint systems and active safety functions made traffic safer than ever before. Latest developments heading from assisted Advanced Driver Assistance System (ADAS) to Automated Driving (AD), lead to more and more complex real-world situations to be handled, going from standard driving tasks up to critical situations, which may cause a collision. Therefore, throughout the development process of such systems, it becomes common to use simulation technologies in order to assess these systems in advance. To gain results out of the simulation, input data are required; typically, from various sources, so the requirements can be covered. Thus, the challenge of scoping with different input sources arises. To come along with that problem, two main kinds of input data will be needed in general: (1) the descriptive parameter covering all border conditions, so called parameter room; (2) the system specifications for estimation. The quality of the results correlates strongly with the quality of inputs given. In case of ADAS systems and AD functions, the second kind of input data is very well known. Major challenges relate to the first kind of input data. Thus, the paper will describe a way to create input data that cover all descriptive parameters needed from normal driving up to the collision by the combination of accident analysis and real-world road traffic observations. The method aims at being applicable to different data sources and to different countries.
\end{abstract}

Key words: ADAS, automated driving, simulation, accident data, traffic observation, database.

\section{Introduction}

The objective is to develop a method that would reconcile accident analysis parameters and real traffic observations, in order to cover the requirements of simulation [1]. Based on an example, the methodology will be explained in the following. There are two main kinds of input data, the accident analysis based on accident databases being the first one, and the observational studies the second one. The accident analysis based on both, data from the on-spot accident investigation called GIDAS (German In-Depth Accident Study [2]) and police reports is performed to distinguish relevant accident parameters and to identify the accident location to be measured. This leads to the second input of data, the real traffic

Corresponding author: Christian T. Erbsmehl, doctor, research fields: vehicle and road safety. observations, which are drawn from a combination of the pre-developed AIMATS-approach (Analysis and Investigation Method for All Traffic Scenarios) along with traffic data classifiers (SDR), applied in an intersection in Germany. With the AIMATS approach it is possible to access parameters such as speed and position from the data of the infrared camera through image processing, based on triangulation [6]. Additionally, the use of the SDR device can provide various information about the traffic (e.g. vehicle speed, vehicle length) based on radar technology. The data acquired provide access to the trajectories of all vehicles which are recorded. By smoothing the data, a trajectory profile is obtained according to the route taken at the measured location with its corresponding parameters. This allows a better understanding of the complexity of situations that lead to accidents. 


\section{Methodology}

2.1 First Input: The Accident Analysis to Identify Hotspots

In order to choose a relevant location to conduct the observation, the first step is to identify hotspots of accidents. An accident analysis is conducted based on GIDAS and on police recorded accident data in Saxony, Germany. The GIDAS data provide detailed accident information such as level of injury, collision details, configuration of the accident scene, while the police records give an overview on real world accident relevance. From this analysis, several possible measurement locations are identified, which need to be further refined. Different criteria are established to filter the results. The selected location shall be a hotspot for accidents resulting in injuries. To be able to study a wide range of manoeuvers, it is advisable to choose a junction instead of a simple road section. The geometry of the junction shall remain simple and the width of the carriageway large enough. Based on these criteria, an X-shape will therefore be chosen. Another selection criterion is the presence of multiple road user types as well as different vehicle types. The chosen junction should then include tramway lines, bicycle paths and pedestrian crossings. These requirements, combined with the authorizations of the various actors in the city and safety recommendations for the installation of equipment lead to the selection of one junction: the intersection between the Kesselsdorfer and the Reisewitzer streets, in Dresden, Saxony. The crossing is X-shaped, with one lane per direction and per arm, except from a preselection lane. The tramway crosses the intersection from east to west, parallel to the bicycle paths, which have their own carriageway. It is regulated by traffic lights and has pedestrian crossing marks. The daily traffic here was estimated between 4.600 and 6.500 vehicles per day in 2016 [3].

\subsection{Second Input: The Real-Life Observations}

Having identified a hotspot for accidents, the next task is to set up the data acquisition system to be able to study vehicles in real-life.

\subsection{Installation}

The different devices are installed on the selected site in order to record various parameters like speed, position, deceleration, and time. To run the AIMATS-method, one infrared camera is set, placed in an opened and cleared space, as well as six SDR devices, whose positions result from the endeavour to measure the entry and the exit of the curve, a compromise between the requirement to access all lanes, and the spatial constraints and actual installation possibilities (Figs. 1

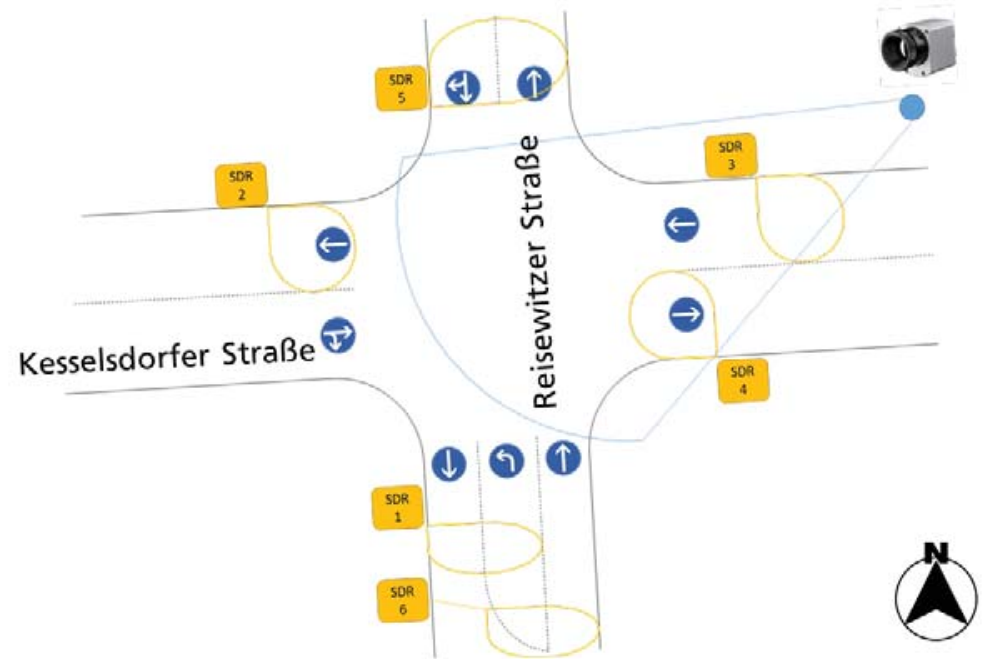

Legend

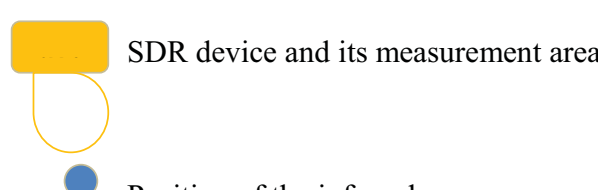

Position of the infrared camera

(1) Direction of travel in a lane

Fig. 1 Positioning the devices on the junction. 

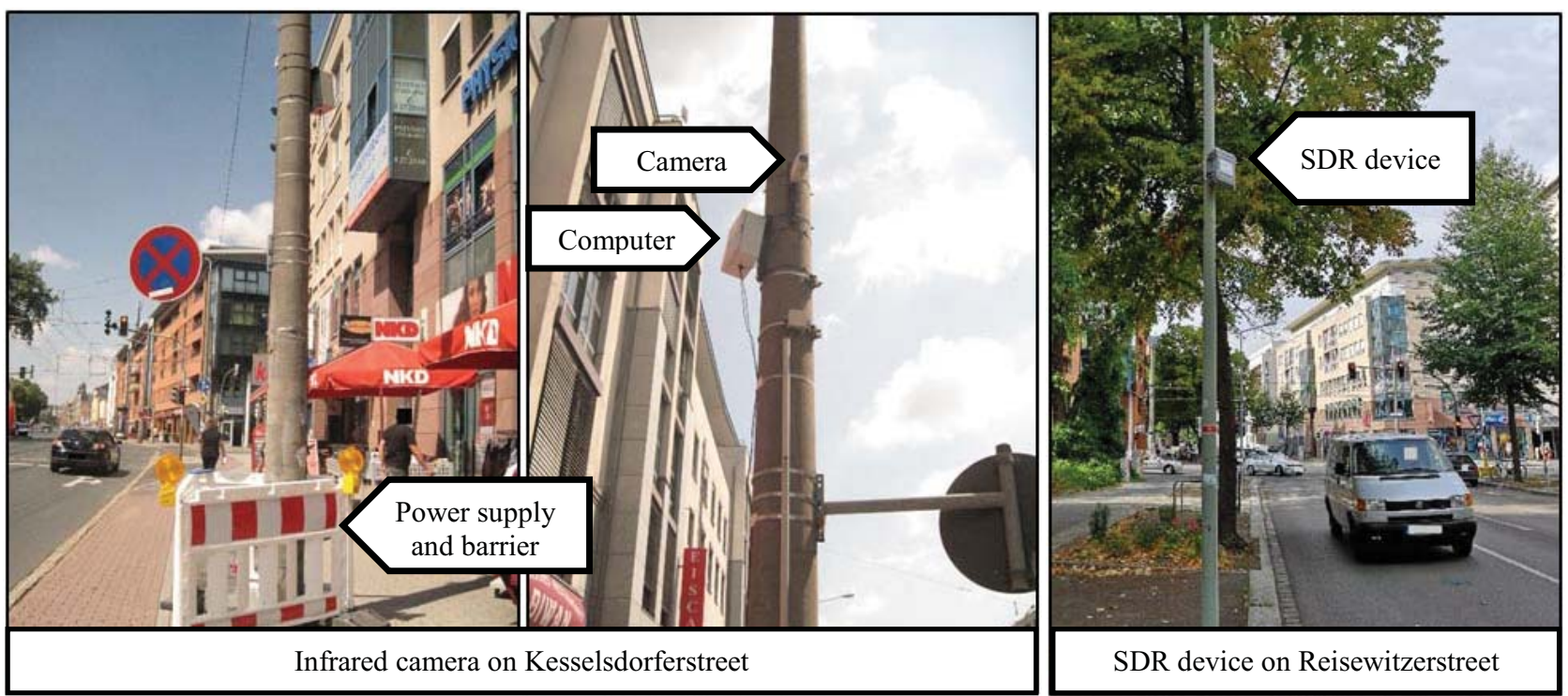

Fig. 2 Measurement equipment installed in the streets.

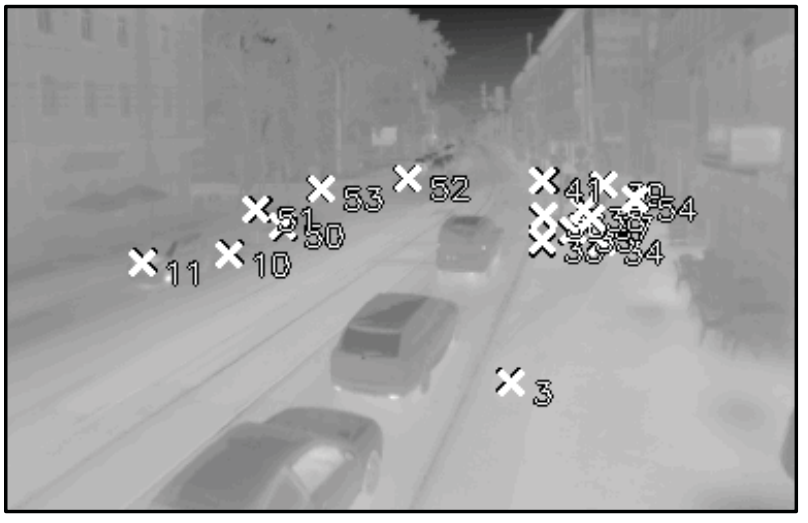

Fig. 3 Position markers on the camera.

and 2). With the infrared camera also comes a computer. A barrier on the sidewalk intends to protect the power installation on the ground (Fig. 2).

\subsection{Measures}

The AIMATS-method is based on the detection of the desired object and its tracking [4]. The infrared camera is able to process position data through an image processing method. Each pixel of the image is mathematically projected onto the street layout in $2 \mathrm{D}$ (Fig. 3), so that the path of a vehicle is known and followed as long as it appears in the vision of the camera. The junction is therefore completely locked down (Fig. 4), in order to identify the different areas of the crossing: the carriageway, the sidewalk, bicycle and tramway lanes.
The result of the analysis of Fig. 4 is a 2D-map version with traffic lane tracking and coordinate system (Fig. 5). Subsequently, if a vehicle is not in an appropriate lane, such as a vehicle climbing onto the sidewalk, it will simply be excluded from the analysis. However, at this crossroad, cars can also drive on tramways.

The installed system started recording in July 2018 for four weeks, day and night. This results in 480 hours of evaluable records.

Additionally, each SDR device collects data like date, time, vehicle class, speed and length of each vehicle detected in its measurement area. Therefore, the SDR data collection method is based on processing the speed of vehicles.

\subsection{Processing Data}

\subsubsection{Trajectories}

To simplify the sorting of data, the data are grouped by nine manoeuvers, which are supposed to be ideal due to the configuration of the crossing, based on the radius of the curve and the driving rules [5]. These nine manoeuvers cover all possible routes depending on the arm of the intersection and the direction. In Fig. 6 , the straight path is shown in blue and the particular section of the curved segment is shown in orange for every manoeuver. 


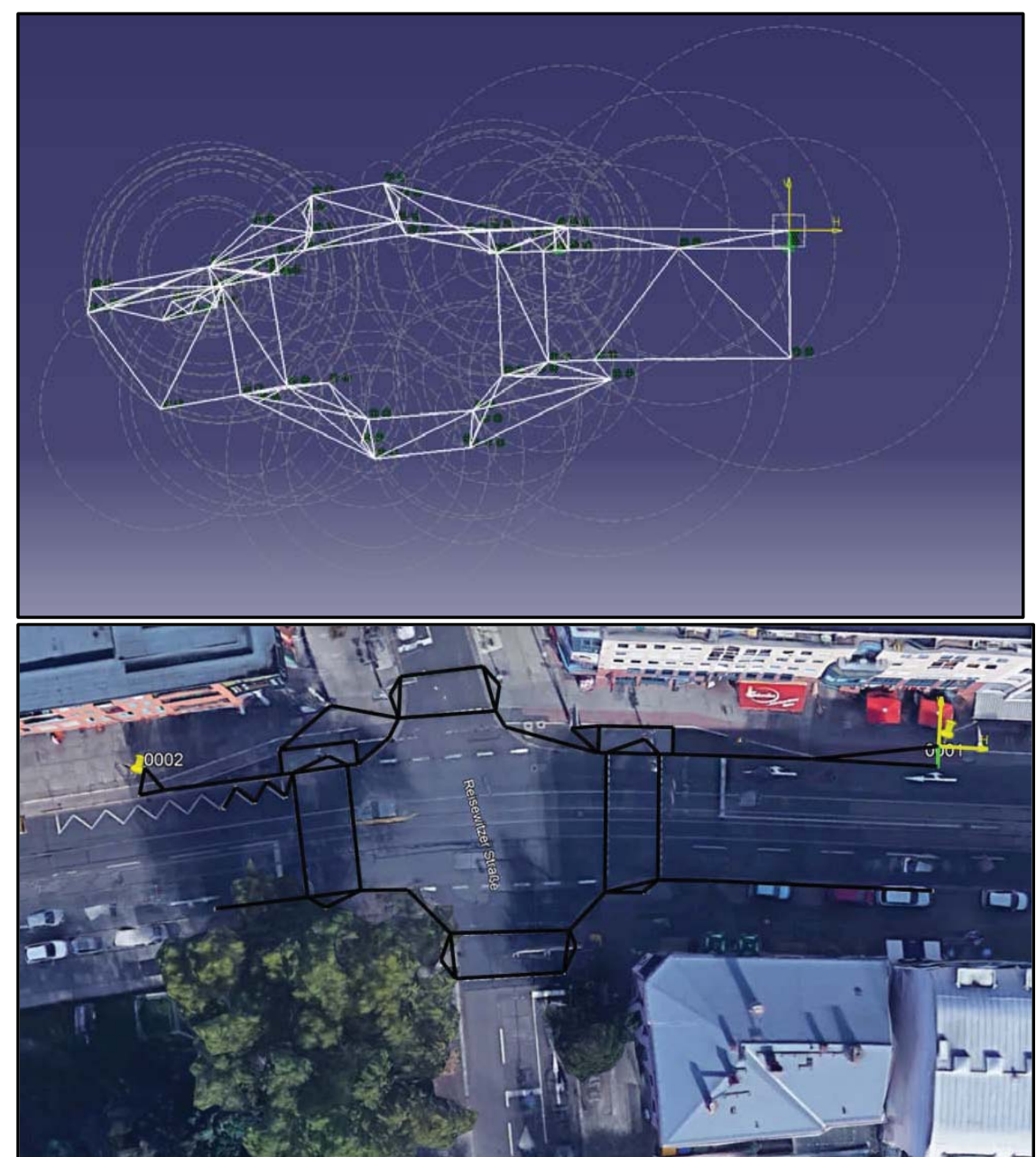

Fig. 4 Projection of markers on the path.

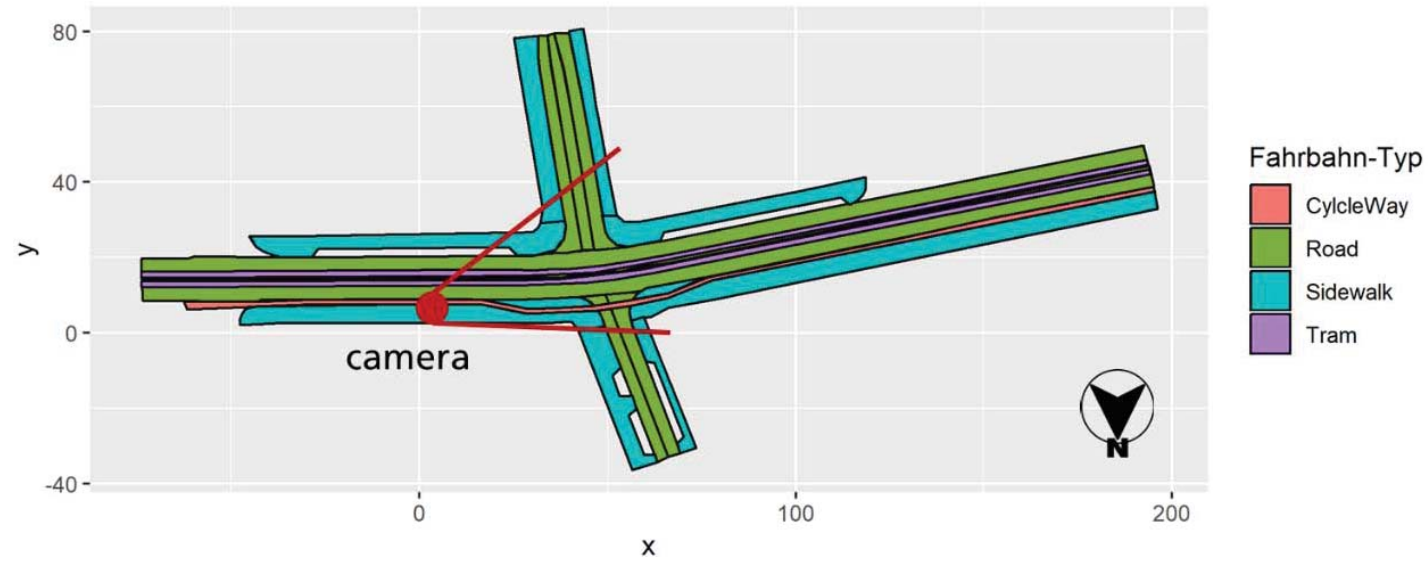

Fig. 5 Illustration of the different lane types in the local coordinate system. 


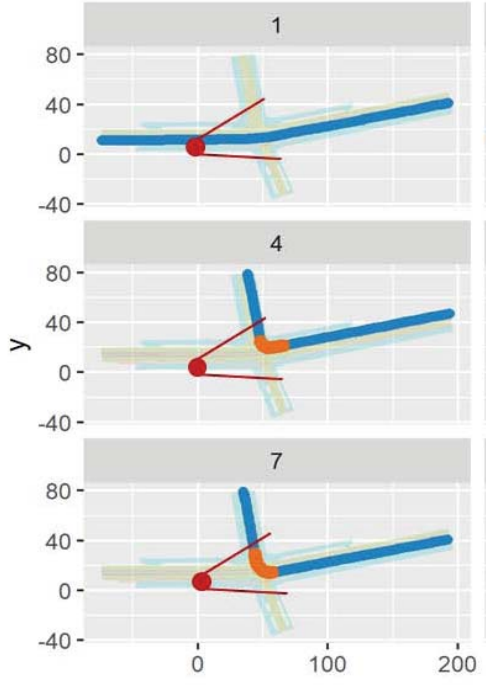

Fig. 6 Definition of the driving manoeuvers for each lane.

A new coordinate system is created, evolving from the original one $(x, y)$ : this mobile coordinate system (s, q) allows dividing different road sections along the ideal manoeuver, with $\mathrm{s}$ along the direction of traveling and $\mathrm{q}$ perpendicular to the direction of travelling. For each section, the distribution of the measured trajectory points is studied. In the mobile system, the points at the two extremities are deleted to avoid extreme values, so that only $90 \%$ of the data are kept. The remaining data are then divided into three subgroups with equal distribution of $30 \%$ : the innermost points of the curve, the points in the middle, and the outmost points. For each of these subgroups, the average is calculated.

The same procedure is applied for each section, which has its own mobile coordinate system. All mean coordinates are known both in the moving coordinate system and in the original one, which is shared. By gathering the values in the original system, the inner, middle and outer trajectories can be drawn (Fig. 7).

\subsubsection{Speed}

The position is not the only information that can be analysed: speed can also be drawn from the information collected using both the AIMATS infrared camera and the SDR devices. For each of the three trajectories, the speed profile is calculated (median and quantile) in the mobile coordinate system along the s-axis (Fig. 8). In the figure, reference 0 is the curve summit and the different road sections are on the ideal trajectory at regular distance intervals (every 5 meters). It is interesting that the further the vehicle moves through the curve, the slower the speed.

SDR devices measure at the start point (SDR 3) and end point (SDR 5) of manoeuvre 3 (see also Fig. 1) throughout the whole measurement period. The collected SDR data provide the basis to generate the two sections at the left and right ends of each graph in Fig. 8. Since the SDR devices do not provide any information in this section of manoeuvre 3, the remaining boxplots derive from AIMATS observations. By combining data of AIMATS and SDR a higher accuracy can be reached to determine speed profiles at entry/exit of manoeuvers.

The data of the SD Rboxplots are derived from the velocity distributions of SDR 3 and 5 (Fig. 9). It is interesting to highlight the higher speed average recorded by SDR 3. However, this device also captures the speed of vehicles going straight ahead and therefore includes data of manoeuver 1 . This could explain a higher average speed, as no deceleration is needed in manoeuvre 1.

\section{Limitations}

It remains hard to cover every type of accident, which would require applying AIMATS method to 


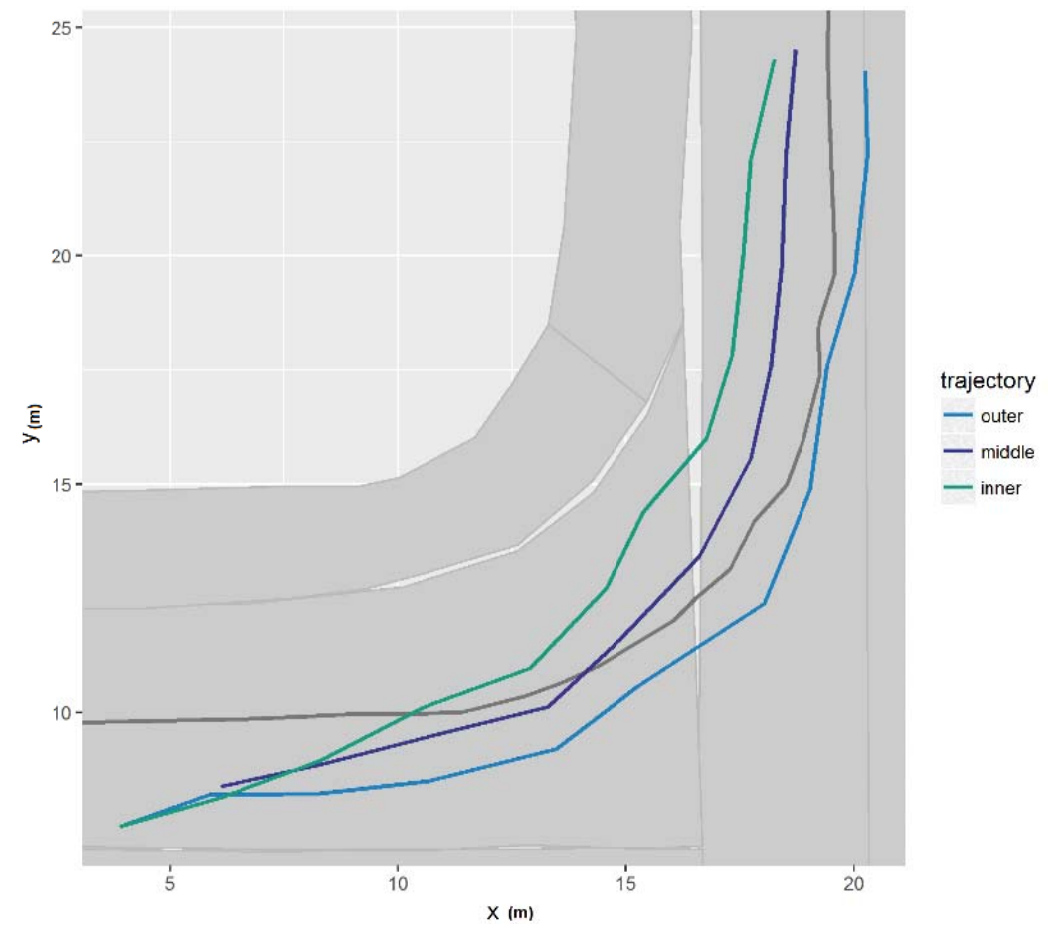

Fig. 7 Example of plot for the outer (blue), middle (purple) and inner (green) trajectories on ideal manoeuver 3.
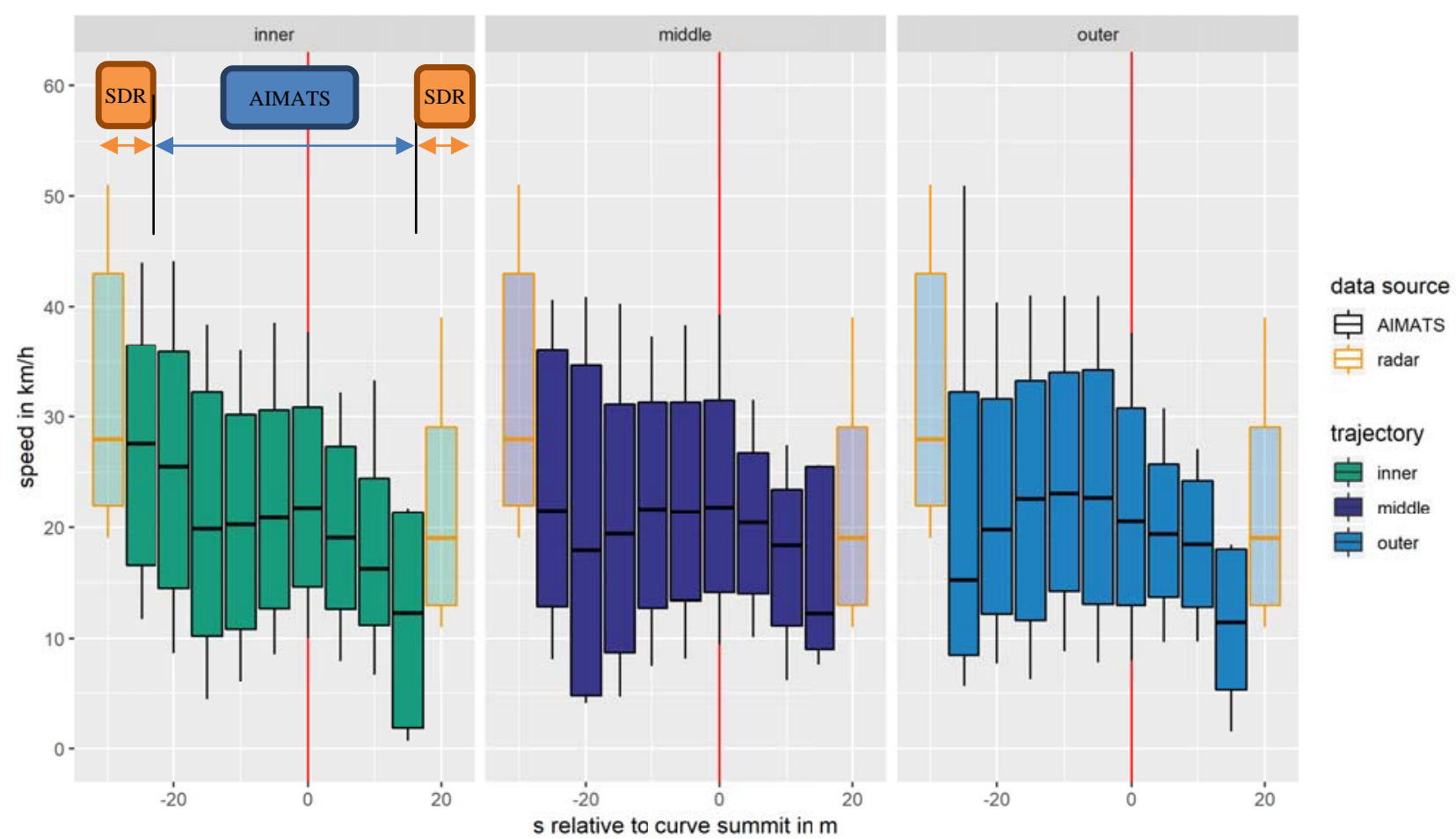

Fig. 8 Distribution of velocity of AIMATS and SDR device 3 and 5 over the daytime. 


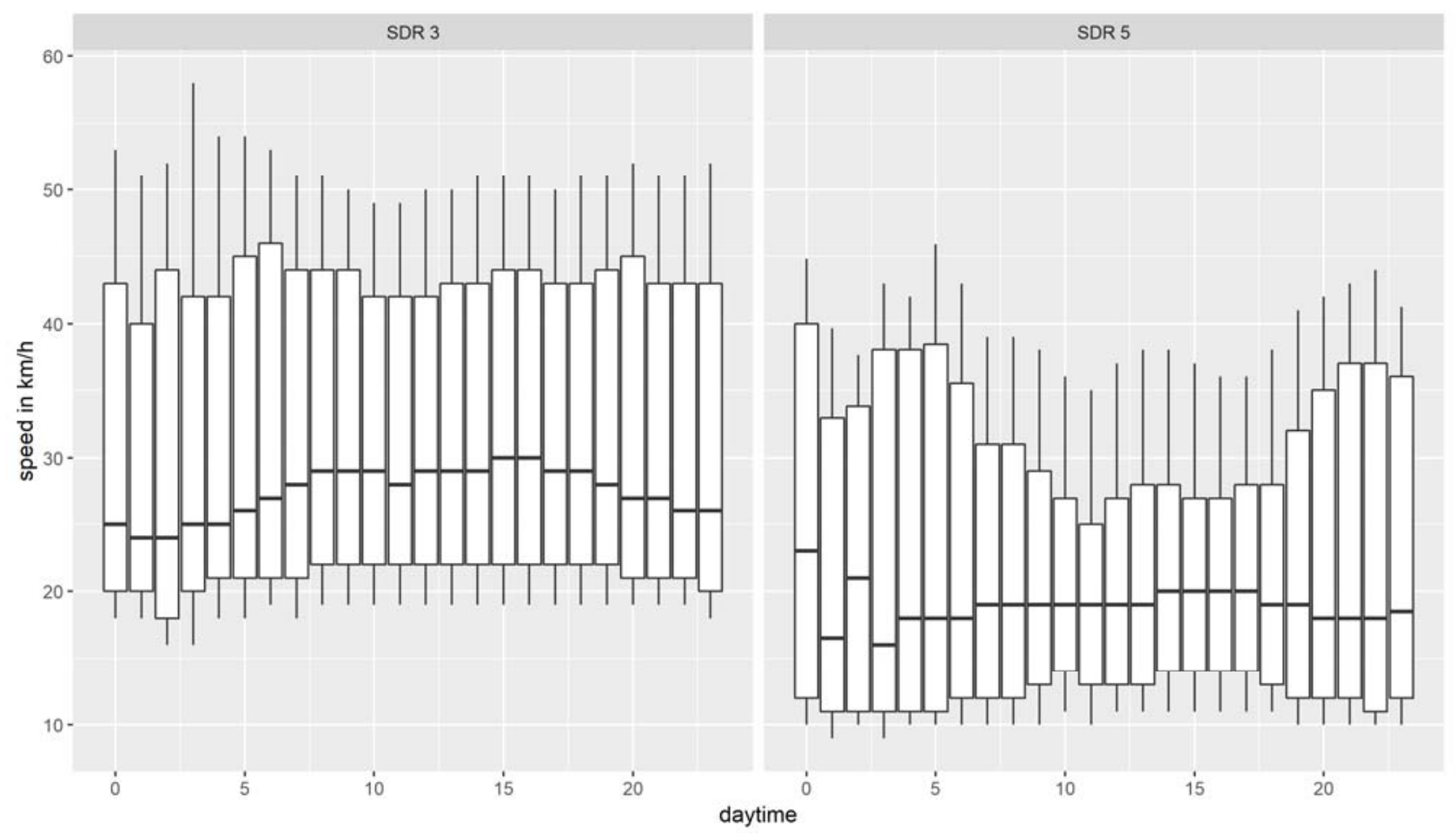

Fig. 9 Speed distribution for SDR cameras 3 and 5 during the day, observing manoeuver 3.

several locations to cover a wide spectrum of accident scenarios. One of the main limitations is indeed, that by studying a location over a defined time range, the rarest scenarios will not be recorded or almost not recorded. To avoid this, it would be necessary to have experience at more crossroads and more arrangements and with more study time: this means more resources and processing time. The resolution of the cameras also limits the study and induces a certain measurement uncertainty. There is definitely room for improvement with regards to image processing. There is also a limit given by the use of the SDR devices: they do not recognize pedestrians and test measurements have shown that cyclists are often not recognized either. Therefore, recognition of different types of vulnerable road users could also be an area for improvements, which could be tackled by using different types of sensor (e.g. LIDAR system).

\section{Conclusion}

The goal was to combine both accident analysis parameters and real-life observations. The accident analysis resulting in two datasets, the GIDAS data and the police record accidents, helped to identify various observation areas and maneuver-based distributions of important parameters for all road users. The AIMATS-approach carried out the real-life observations along with SDR device. The real-world driving behavior and maneuver is measured. The speed profile and other valuable information can be determined using the measurements to support the simulation of real-world road traffic behaviors. For example, mean speed or mean deceleration of different maneuvers e.g. turning or crossing can be derived from the data. These measurements further will be used to create descriptive parameters supporting driver behavior models.

This publication will show a further step to generate reliable large-scale simulation basis files for the assessment of ADAS and AD, which are based on real world accidents and traffic observation measurements. The method can also be applied in different countries even under different circumstances. 


\section{References}

[1] Rosén, E. 2013. "Description of AEB System Parameters." In Autonomous Emergency Braking for Vulnerable Road Users. Gothenburg: IRCOBI, pp. 618-27.

[2] GIDAS. "GIDAS Homepage." Available: www.gidas.org.

[3] Landeshauptstadt Dresden. 2016. “Traffic Volume in Vehicle per Day.” Dresden.

[4] Landgraf, T., Erbsmehl, C. T., and Yuasa, H. 2017. "An In-Depth Study for the Investigation of
Intersection Accidents Using the AIMATS-Method.” In FAST-Zero'17 Future Active Sefety Technology towards Zero Traffic Accident, Nara Kasugano, Japan.

[5] Bundesministerium der Justiz und für Verbraucherschutz, Straßenverkehrs-Zulassungs-Ordnung, Berlin, 2019.

[6] Landgraf, T., Erbsmehl, C. T., and Yuasa, H. 2015. "In-Depth Field Study of Animal Street Crossing Behaviour.” Presented at ESV Conference, 25th International Technical Conference on the Enhanced Safety of Vehicles, Detroit, USA. 